\title{
DESARROLLO Y CONSTRUCCIÓN DE UN VEHÍcULO AÉREO NO TRIPULADO APLICADO A LOS INCENDIOS FORESTALES
}

\section{DEVELOPMENT AND CONSTRUCTION OF AN UNMANNED AERIAL VEHICLE FOR WILDFIRE MANAGEMENT}

José Daniel Lencinas ${ }^{1}$ Ezequiel Parodi ${ }^{2}$ Brigitte Van den Heede ${ }^{3}$ Luciana Heitzmann ${ }^{4}$

1. Dr. en Ciencias Forestales, especialista en geomática. Director Ejecutivo del CIEFAP (Centro de Investigación y Extensión Forestal Andino Patagónico), Sede Esquel, Provincia del Chubut, (Argentina). E-mail: jdlencinas@ciefap.org.ar

2. Lic.marketing. Gerente de Life Style Media, Trevelin, Provincia del Chubut, (Argentina). E-mail: queque.parodi@gmail.com

3. M.Sc. Gestión y Auditorías Ambientales. Coordinadora, Proyecto Estratégico del CIEFAP: "Desarrollo e Innovación tecnológica para el monitoreo de catástrofes" -P7.A2.003-.CIEFAP (Centro de Investigación y Extensión Forestal Andino Patagónico), Sede Esquel, Provincia del Chubut, (Argentina). E-mail: bvandenheede@ciefap.org.ar

4. Lic. Biología. Tecnóloga contratada por CIEFAP (Centro de Investigación y Extensión Forestal Andino Patagónico), Sede Esquel, Provincia del Chubut, (Argentina). E-mail: heitzmann.luciana@gmail.com 


\section{RESUMEN}

Los incendios forestales en Patagonia son el factor principal de pérdida de superficies boscosas. Es fundamental optimizar y mejorar el actual sistema de manejo del fuego desde su prevención, sistemas de alerta temprana, y el combate propiamente dicho. En este marco, el Centro de Investigación y Extensión Forestal Andino Patagónico (CIEFAP), en un entorno público privado, desarrolló un vehículo aéreo no tripulado, denominado Fénix $3 \mathrm{~d}$, como herramienta complementaria dentro del sistema de manejo del fuego.

\section{ABSTRACT}

Forest fires in Patagonia are the main factor of loss of forest areas. It is essential to optimize and improve the current system of fire management from prevention, early warning systems, combat itself. In this framework the Patagonian Andes Research and Extension Center (CIEFAP), in a private and public environment, developed an unmanned aerial vehicle, called Fénix $3 \mathrm{~d}$, as complementary tool within a fire management system.

\section{PALABRAS CLAVE}

Fénix 3d, VANT, Incendios Forestales, Cuadricóptero, Patagonia.

\section{KEY WORDS}

Fénix 3d, UAV, Wildfires, Quadcopter, Patagonia. 


\section{INTRODUCCIÓN}

Se estima que los incendios forestales globales consumen 9.000 millones de toneladas de biomasa vegetal y que son responsables del 30\% de las emisiones de dióxido de carbono (Bowman et al., 2009). En relación a los bosques templados fríos de Patagonia, los incendios forestales son el factor principal de pérdida y degradación de superficies boscosas. Todos los años en la época estival se incrementa el número de focos de incendios. Como valor referencial en cuanto a las frecuencias, en el año 2014 se registraron en la región patagónica 1.092 focos de incendios, la mayoría producidos por causas antrópicas (MAyDS, 2016). El promedio anual de bosques nativos afectados por fuego en Patagonia en el período 2001-2012, fue de 3.500 ha. Sin embargo, considerando las dos últimas temporadas de verano $2013 / 2014$ y 2014/2015 los incendios afectaron 47.378 ha, de las cuales el $60 \%$ corresponde a áreas de bosque alto (MohrBell, 2015). En la temporada estival 2015/2016 se incendiaron más de 20.000 ha. Los incendios en áreas de interfase, bosque-población, no solo afectan bosques, sino también infraestructura, viviendas y ponen en riesgo vidas humanas. Se estima que para el combate de un incendio forestal de gran magnitud ( $>500$ ha) en la interfase en Patagonia, el estado eroga más de USD 100.000 al día, considerando la utilización de medios terrestres y aéreos tales como aviones hidrantes y helicópteros con helibaldes. Este valor es de referencia ya que depende de la accesibilidad, cantidad de viviendas e infraestructura a proteger. Estos montos son comparables a los incendios forestales en países como Italia y España (Ortuño Pérez y Sigfredo, 2007).

\section{TECCNOLOGÍAS APLICADAS EN LAS DIFERENTES FASES DE LOS INCENDIOS FORESTALES}

El tipo de tecnologías utilizadas se puede diferenciar de acuerdo a las tres fases de gestión de los incendios forestales:

a- Antes del incendio, (sistema de pronóstico del grado de peligro de ocurrencia, sistemas de detección de incendios forestales, i.e monitoreo preventivo).

b- Durante el incendio (detección de focos activos, acciones de extinción o supresión, planificación de evacuación y de rescate).

c- Posterior al incendio (evaluación de daños y procesos de restauración ecológica post-fuego).

En relación a la primera fase, existen diferentes tecnologías, las más utilizadas son las terrenas, así como los relevamientos aéreos y satelital. Los dispositivos terrestres que están cobrando relevancia se conforman de un sistema de torres con cámaras térmicas y ópticas formando una red fija de vigilancia que transmite en tiempo real imágenes a un centro de control. Las torres se emplazan en puntos estratégicos de la topografía, y se dotan de cámaras duales (visión térmica y visión óptica). Realizan el monitoreo perimetral a 360ํ, en forma continua, con una visión de hasta $20 \mathrm{~km}$ a la redonda cada una.

Respecto a la tecnología satelital, en las últimas décadas se han desarrollado nuevas aplicaciones a nivel operativo para las tres fases mencionadas. El rango de sensores y de técnicas empleados es muy amplio, y se basan en la medición de la energía liberada por los incendios. Se debe considerar que durante grandes incendios forestales se registran temperaturas extremas entre 600-1000 ㅇ en la 
parte superior del dosel. Sun (2007), determinó dos picos de emisión en la banda del infrarrojo medio, uno de ellos a 4,17 $\mu \mathrm{m}$ que refleja el máximo de emisión de CO y el otro se extiende a través de las longitudes de onda de 4,34 $\mu \mathrm{m}$ a 4,76 $\mu \mathrm{m}$. Sobre esta base de información, las plataformas satelitales que se utilizan para la detección de puntos calientes, por lo general, detectan la energía liberada en el infrarrojo medio (MIR: 3-5 $\mu \mathrm{m}$ ), infrarrojo térmico (TIR; 8-14 $\mu \mathrm{m}$ ) que provee la temperatura del suelo, e infrarrojo cercano (SWIR:1.0-2.5 $\mu \mathrm{m}$ ). Además, los algoritmos desarrollados se complementan con la información de bandas correspondientes al sector visible. A nivel global, el satélite MODIS (Moderate Resolution Imaging Spectroradiometer) es sin dudas el sensor más utilizado para la detección de puntos calientes (Giglio et al., 2016). Sin embargo, tanto las plataformas satelitales con alta resolución temporal como MODIS, así como otras plataformas geoestacionarias o de satélites de baja órbita presentan serias limitaciones especialmente por su moderada resolución espacial para detectar el inicio de focos de incendios (Alkhatib, 2014). Es por ello que una de las líneas de desarrollo europeas está enfocada al diseño y construcción de microsatélites como el Fire Monitoring Constellation (FMC) con cámaras ópticas y térmicas de mayor resolución espacial. Es el caso del micro satélite TET-1 (Technologie Erprobungs Träger-1) y el reciente lanzamiento del BIROS (Berlin Infrared Optical System) para que actúe en tándem con el TET-1 (http://www.dlr.de/).

Finalmente, la detección por medios aéreos, tiene dos vertientes tecnológicas, la tradicional tripulada, mediante la operación de aviones y helicópteros, y la tecnología de vehículos aéreos no tripulados (Merino et al. 2012).

La detección temprana de focos de incendios es sin duda a nivel global la variable más importante para reducir costos y evitar pérdidas. Esto implica sistemas de resolución temporal y espacial con eficientes sistemas de comunicación, combinación de cámaras ópticas y térmicas para capturar imágenes y detectar puntos calientes independientemente de la cobertura del dosel y de las condiciones de luminosidad natural. En este contexto, una línea de vanguardia en innovación tecnológica se basa en la construcción de vehículos aéreos no tripulados (VANT o UAV del término en inglés Unmanned Aerial Vehicle) denominados comúnmente drones, como complemento tecnológico a sensores terrestres o de plataformas aerotransportadas (aviones, helicópteros) y satelitales. Para su efectividad, tal tecnología debería tener fácil adopción por el usuario y adaptación al terreno en el cual ocurren los eventos: estar asociada directamente a los sistemas organizacionales que intervienen en las catástrofes (Van den Heede, 2015).

Bajo esta premisa y con el fin de desarrollar tecnologías para las dos primeras fases de incendios en bosques de montaña se desarrolló el VANT Fénix 3d, cuyas características y desempeño se describen a continuación.

\section{DISEÑO Y CONSTRUCCIÓN DEL CUADRICÓPTERO}

El vehículo aéreo no tripulado (VANT) denominado Fénix 3d es un cuadricóptero desarrollado en un entorno público-privado (CIEFAP -Life style media) especialmente para actuar en eventos de riesgo tales como incendios o erupciones volcánicas. El Fénix $3 d$ está desarrollado en base a tecnología multirotor y parte de sus componentes se diseñaron y construyeron con impresora 3D (Gráfico 1). 


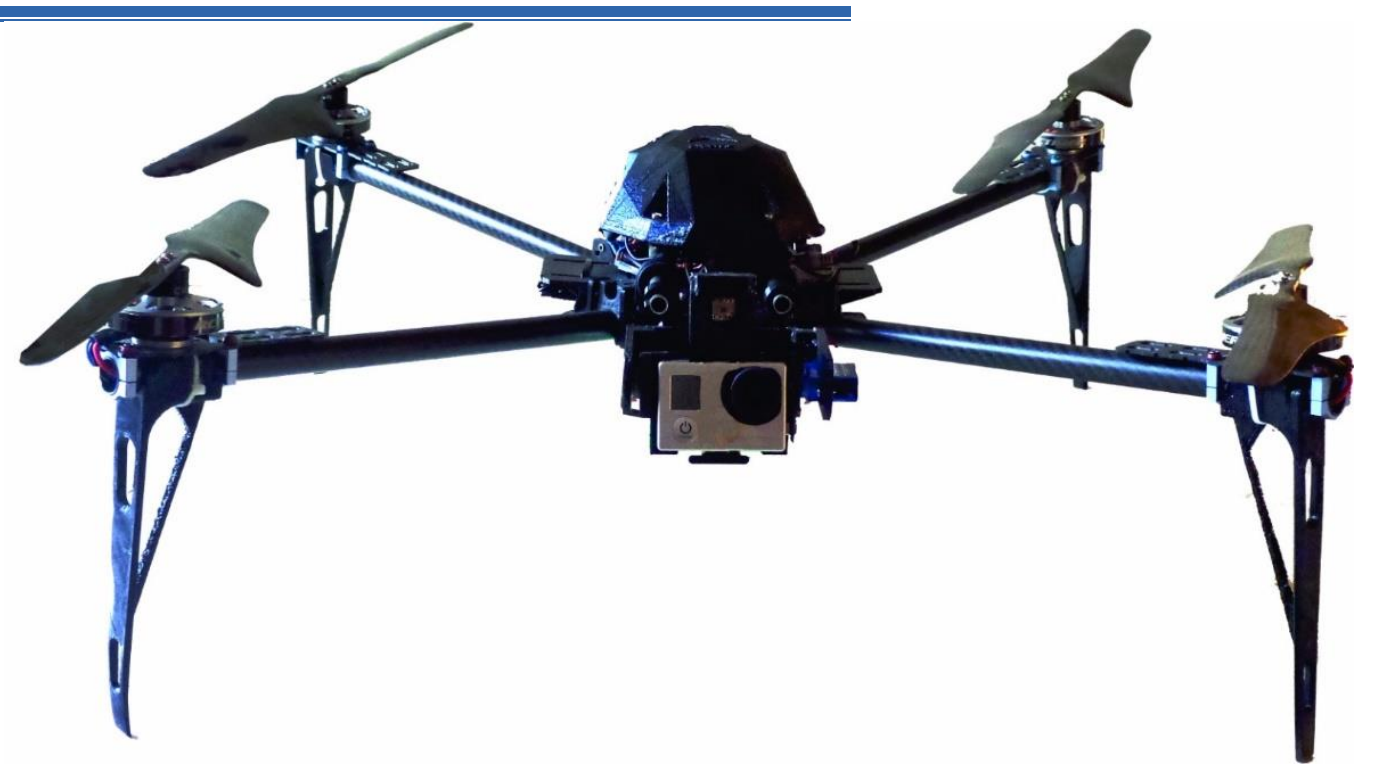

Gráfico 1: Imagen real del cuadricóptero Fénix 3d.

Fuente: elaboración propia.

El vehículo está controlado remotamente mediante un radio control. Posee un chasis ultraligero de fibra de carbono y su diseño plegable es importante para su transportabilidad, considerando que los incendios forestales en la cordillera de los Andes. Generalmente, se presentan en áreas de baja accesibilidad por su topografía escarpada y baja densidad de red de caminos.

\section{A- Componentes}

Una de las variables críticas de los VANTs es su autonomía de vuelo. Con el fin incrementarla, en el Fénix 3D se realizaron desarrollos de integración y optimización de componentes para minimizar su peso. A continuación se presentan en la Tabla 1 las características principales del cuadricóptero construido.

Tabla 1. Características principales del cuadricóptero Fénix 3d.

\begin{tabular}{|c|c|}
\hline Chasis/Tamaño diagonal & fibra de carbón / $180 \mathrm{~mm}$ plegado-600 mm extendido \\
\hline Peso total del VANT/ Rotores & $2,450 \mathrm{~kg} / 17 \times 5,5$ pulgadas \\
\hline Cubierta & ABS impresa con tecnología 3D \\
\hline Landing & desmontable de ABS impreso con tecnología 3D \\
\hline Sistema de navegación & GPS. \\
\hline Batería & Polímero de litio, 4 celdas, $10.000 \mathrm{mAh}$ \\
\hline Controlador / Radio control & DJI / Turnigy 9xr con Sistema long Rage \\
\hline Frecuencia de Operación & $2,4 \mathrm{GHz}$ vuelo autónomo, 1,2 ghz video, $433 \mathrm{mhz}$ radio \\
& control \\
\hline
\end{tabular}

Fuente: elaboración propia.

El multi-rotor transporta una cámara térmica y una cámara óptica integradas. La cámara térmica es sensible al infrarrojo térmico de onda larga (Longwave Infrared LWIR) de FLIR Lepton ${ }^{\circledR}$ (Tabla 2). Detecta energía en una banda y su resolución espectral se encuentra en el rango de $8 \mu \mathrm{m}$ a $14 \mu \mathrm{m}$. El tipo de lente es $\mathrm{f} / 1.1$ silicon doublet. 
Tabla 2: Especificaciones principales de la cámara LWIR integrada al Fenix 3D.

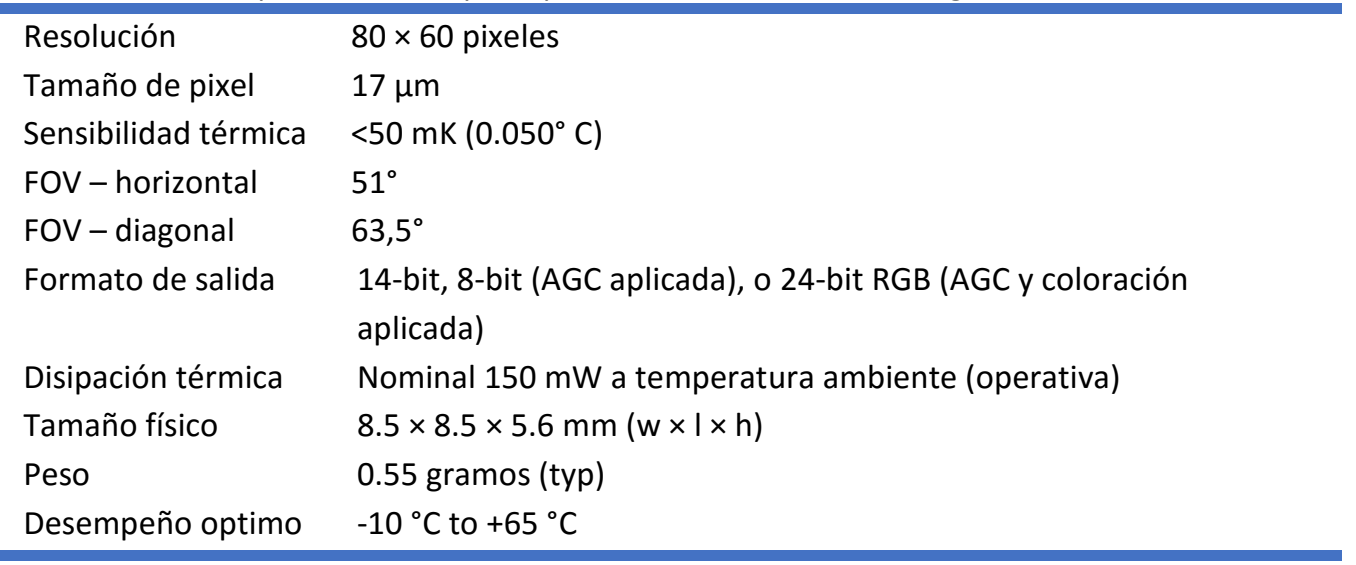

Fuente: elaboración propia.

La cámara óptica se trata de una cámara GoPro, lente óptico F2.8 - 6 elementos, lente ultra gran angular, con una resolución de 640×490/720x1280 / 1920×108 para modos gran angular/medio/ estrecho respectivamente, y un formato de salida MP4.

La cámara GoPro se alimenta de la batería Li-Po del propio VANT. Los videos se transmiten en tiempo real a una estación en tierra y se pueden grabar en la tarjeta micro SD de la GoPro. Las imágenes de la cámara térmica también se trasmiten en tiempo real. Se puede conmutar de óptica a térmica y térmica a óptica.

\section{B- Fase de Pruebas}

Desde su concepción, en la ingeniería de detalle, y análisis de calidad, se tuvieron en cuenta las necesidades de los usuarios. Una vez construido el Fénix $3 d$ fue sometido a diferentes pruebas en cinco niveles:

- Nivel 1: Pruebas de laboratorio

- Nivel 2: Pruebas de vuelo en ambientes controlados

- Nivel 3: Pruebas de resistencia ante impactos

- Nivel 4: Pruebas en ambientes no controlados

- Nivel 5: Pruebas en Incendios Forestales

La información de la Tabla 3 surge luego de haber superado las cinco fases de prueba. En la fase cinco, el VANT Fénix $3 d$ fue utilizado en un gran incendio forestal ocurrido en el Parque Nacional Los Alerces en la Provincia del Chubut, localizado en la Patagonia Argentina. El incendio se inició el 21 de enero y afectó aproximadamente 1.700ha. En la Tabla 3 se presentan valores de referencia, los cuales fueron determinados en todas las fases de control de calidad y en el evento extremo, con vientos intensos con ráfagas de hasta $60 \mathrm{~km} / \mathrm{h}$.

Tabla 3: Características del Fénix 3d en vuelo.

\begin{tabular}{cc}
\hline Fénix $3 \mathrm{~d}$ & Performance \\
\hline Precisión de vuelo estacionario & Vertical: $0,5 \mathrm{~m} /$ Horizontal: $2,5 \mathrm{~m}$ \\
Velocidad angular máxima & $10 \mathrm{~m} / \mathrm{s}$ \\
Máximo ángulo de ataque & $35^{\circ}$ \\
Máxima velocidad de ascenso & $10 \mathrm{~m} / \mathrm{s}$ \\
\hline
\end{tabular}


Máxima velocidad de descenso

Máxima altura permitida

Máxima velocidad

Resistencia al viento

Tiempo máximo de vuelo

Temperatura operativa

Distancia operativa (alcance radio control )

Distancia de cobertura de video en tiempo real
Área de Innovación y Desarrollo, S.L.

ISSN: $2254-4143$

DOI: http://dx.doi.org/10.17993/3ctecno.2016.v5n4e20.27-35

$$
4 \mathrm{~m} / \mathrm{s}
$$

$1200 \mathrm{~m} \mathrm{~s} /$ punto de despegue

$18 \mathrm{~m} / \mathrm{s}$ (modo ATTI, sin viento)

$45 \mathrm{~km} / \mathrm{h}$ hasta $60 \mathrm{~km} / \mathrm{h}$

$42 \mathrm{~min}$

$14^{\circ}$ a $104^{\circ} \mathrm{F}\left(-10^{\circ}\right.$ to $\left.40^{\circ} \mathrm{C}\right)$

$25 \mathrm{~km}$

$5 \mathrm{~km}-7 \mathrm{~km}$

Fuente: elaboración propia.

\section{C- Modos de vuelo}

Las aplicaciones reales del Fénix $3 \mathrm{~d}$ para tareas relacionadas a la prevención, manejo y monitoreo de los incendios fueron las siguientes:

Vuelo modo torre: El operador eleva el VANT en dirección vertical a una altura $200 \mathrm{~m}$ y gira $360^{\circ}$. Este modo emula a los observadores de una torre terrestre de control. En $200 \mathrm{~m}$ sobre el suelo, se determinaron los siguientes parámetros, que pueden ser tomados como referencia para la cámara óptica en tres configuraciones del lente, ya que se debe considerar las fuertes distorsiones geométricas que ésta produce.

- Gran angular (14 mm FOV): 45km de radio de visión panorámica, Dependiendo de la topografía, hasta un radio de $1,5 \mathrm{~km}$, la resolución espacial relativa es mayor a $2 \mathrm{~m}$.

- Medio (21 mm FOV): $22 \mathrm{~km}$ de radio de visión panorámica, dependiendo de la topografía, hasta un radio de $1,25 \mathrm{~km}$, la resolución espacial relativa es mayor a $3 \mathrm{~m}$.

- Estrecho ( $28 \mathrm{~mm}$ FOV): $600 \mathrm{~m}$ de radio de visión panorámica, dependiendo de la topografía, en toda la superficie capturada, la resolución espacial relativa es mayor a $1 \mathrm{~m}$ (i.e. submétrica).

Vuelo detección de puntos calientes: En este modo se utiliza la cámara óptica y se alterna con la visualización de las imágenes capturadas con la cámara térmica, con el fin de detectar puntos calientes. Los datos capturados por el sensor térmico de la cámara, no son afectados por el humo o por la estructura del dosel.

Vuelo modo reconocimiento: El operador programa o pilotea el VANT por las zonas de interés. Por ejemplo, el Fénix $3 d$ se utilizó para la determinación de todo el perímetro del incendio, con el fin de tomar medidas de control terrestre, orientadas a resguardar el avance del incendio o detectar nuevos focos. Además es importante para estimar la cantidad de recursos terrestres que se deben desplegar, para proteger los sitios más sensibles del perímetro y cuantificar diariamente toda la superficie afectada por el incendio.

Vuelo modo búsqueda: Este modo se utilizó para la búsqueda de personas que se encuentran en la zona del incendio, con el fin de tener documentación objetiva para la investigación sobre las posibles causantes del incendio. Por otro lado, la cámara térmica pudo detectar durante esta prueba, a los brigadistas debajo del dosel del bosque, con el VANT elevado a una altura de $50 \mathrm{~m}$ sobre el dosel. 


\section{CONCLUSIONES}

EI VANT Fénix 3D demostró una alta performance en eventos extremos y se posiciona como un VANT competitivo a nivel internacional. Las imágenes obtenidas son valiosos aportes para las investigaciones sobre la predicción de la propagación del fuego. El VANT proporciona una vista tridimensional, volando relativamente cerca de las columnas de incendios, suministrando información difícilmente observable con medios terrestres. Pueden complementar los medios aéreos, mediante vuelos de reconocimiento y observaciones de comportamiento, sin necesidad de destinar horas de vuelo de helicópteros o aviones hidrantes para esta tarea.

Las ventajas comparativas del Fénix 3d en la etapa de observación del incendio son las siguientes:

- Bajo costo: el valor del Fénix 3d es similar a 23 horas de vuelo de helicóptero.

- Flexibilidad: Fénix 3d puede volar estacionario en las cercanías de un incendio sin que el operador corra riesgos y transmitir en tiempo real los puntos de interés del operador. Maniobrar un helicóptero en estas situaciones puede ser de alto riesgo. Además permite el vuelo nocturno con su cámara térmica mientras las aeronaves tripuladas en Patagonia deben suspender sus vuelos en los lapsos de oscuridad (humo denso/noche).

- Disponibilidad: en incendios forestales las acciones deben ponerse en marcha en cortos plazos de tiempo, el despliegue del VANT Fenix 3d como herramienta, es prácticamente inmediato. Esto permite acceder a información de la situación de la emergencia en pocos minutos, entre un $1 \%$ y un $5 \%$ del tiempo que tarda una aeronave tripulada en poder sobrevolar la zona.

- Sin intrusión: el VANT no tiene efecto sobre el fuego en las acciones de monitoreo, mientras que un helicóptero puede producir ráfagas descendentes que pueden afectar el comportamiento de las llamas.

En síntesis, el vehículo aéreo no tripulado Fénix 3d está desarrollado en base a tecnología multirotor y presenta aspectos innovadores. Es controlado remotamente mediante un radio control. Su chasis ultraligero de fibra de carbono lo convierte en uno de los más livianos y resistentes del mercado, considerando su capacidad de carga y estabilidad de vuelo hasta con vientos de $40 \mathrm{~km} / \mathrm{h}$ y soportando ráfagas de viento de hasta $60 \mathrm{~km} / \mathrm{h}$. Además, su diseño plegable facilita su transporte. Futuros desarrollos en cámaras térmicas en cuanto a resolución espacial, espectral y radiométrica, podrían mejorar las prestaciones del Fénix $3 d$. Su puesta en funcionamiento en diferentes modos y en plena coordinación dentro del sistema de manejo del fuego mediante protocolos aprobados, contribuirá a mejorar la eficiencia de la prevención y supresión de los incendios forestales.

\section{REFERENCIAS BIBLIOGRÁFICAS}

Ahmad A. A. Alkhatib. (2014). A Review on Forest Fire Detection Techniques. International Journal of Distributed Sensor Networks. Volume 2014, Art. ID 597368, 12 pages.

Bowman, D, Balch, JK, Artaxo, P, Bond, W, Carlson, J, Cochrange, MA, D'Antonio, CM, DeFries, RS, Doyle, JC, Harrison, SP, Johnson, FH, Keeley., JE, Krawchuk, MA, Kull, CA, Marston, JB, Moritz, 
MA, Prentice, IC, Roos, Cl, Scott, A, Swetnam, TW, van der Werf, GR y Pyne, SJ. (2009). Fire in the Earth System. Science, 324, pp. 481 - 484., 10.1126/science.1163886.

Giglio L., Schroeder, W., y Justice, C.O. (2016). The collection 6 MODIS active fire detection algorithm and fire products. Remote Sensing of Environment, 178, 31-41.

Merino L., Caballero, F., Martínez-de-Dios, J.R., Maza, I., Ollero, A. (2012). An Unmanned Aircraft System for Automatic Forest Fire Monitoring and Measurement. J. Intell Robot Syst. 65: 533.

Ministerio Ambiente y Desarrollo Sustentable. (2016). Estadísticas de Incendios Forestales. Recuperado de: <http://www.ambiente.gov.ar/>.

MohrBell D. (2015). Superficies afectadas por incendios en la región Bosque Andino Patagónico durante los veranos de 2013-2014 y 2014-2015. Disponible en: $<$ http://www.ciefap.org.ar/index.php?option=com content\&view=category\&layout=blog\&id= $\underline{221 />}$.

Ortuño Pérez y Sigfredo F. (2007). Evaluación económica de daños causados por incendios forestales. IV Conferencia internacional sobre Incendios forestales. Sevilla, España. <http://www.fire.unifreiburg.de/sevilla-2007/contributions/html/in/sTem 1.html/>.

Sun, J. y Xiong X. (2007). MODIS Polarization Sensitivity Analysis. IEEE Trans. Geosci. Rem. Sens., vol. 45, no.9: $2875-2885$.

Van den Heede, B. (2015). Desarrollo e Innovación tecnológica para el monitoreo de catástrofes. Informe Técnico de avance. Disponible en: $<$ http://www.ciefap.org.ar/index.php/component/phocadownload/category/20-programa-7investigacion-y-desarrollo-de-caracter-estrategico/>.

\section{AGRADECIMIENTOS}

Agradecemos al Ministerio de Ciencia, Tecnología e Innovación Productiva de la Nación Argentina por el financiamiento otorgado para la ejecución del Proyecto Estratégico del CIEFAP: "Desarrollo e Innovación tecnológica para el monitoreo de catástrofes" /P7.A2.003. 